\title{
NREL Algal Biofuels Projects and Partnerships
}

\section{NREL is engaged in several algal biofuels research and development projects focused on improving the economics of the algal biofuels production process}

NREL is involved in several algal biofuels projects with national and international partners funded through the U.S. Department of Energy's (DOE's) Bioenergy Technologies Office and NREL's Laboratory Directed Research and Development (LDRD) program. These projects are addressing the challenges that accompany the algal biofuels production process.

Microalgae-photosynthetic microorganisms capable of converting atmospheric $\mathrm{CO}_{2}$ to biomass and oil-offer great promise to contribute a

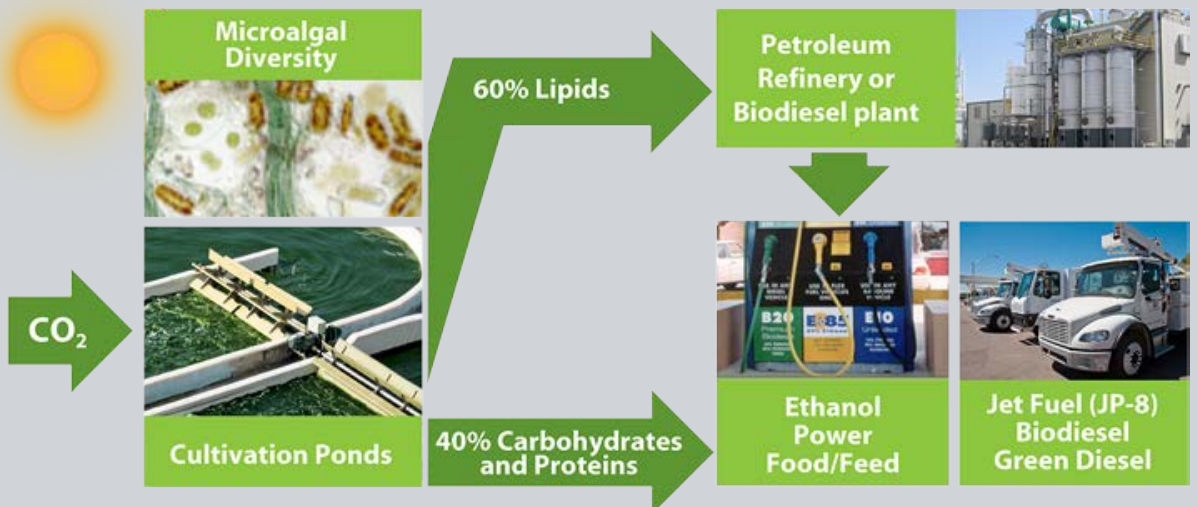

Summary of the current algal biomass-to-biofuels product streams. significant portion to our renewable fuels and meet the mandate to dramatically reduce our dependence on the world's dwindling natural energy resources.

Despite its huge potential, the technology of using microalgae as biomass feedstocks for advanced biofuels faces major challenges from both technical and economic barriers. These include developing suitable algal strains and cultivation parameters, developing processes to harvest the biomass and extract the oils, and rendering the entire process economical.

NREL's current algal biofuels program builds on the expertise and knowledge acquired from the research into microalgal biofuels done by the Aquatic Species Program, which was established in 1978 under funding from DOE.

Novel Microalgal Production and Downstream

Processing Technologies for Alternative

Biofuels Applications

NREL is investigating alternative conversion pathways to obtain the most value from all biofuel-relevant components in algal biomass. A novel fractionation process allows for recovery of separate lipid, carbohydrate, and protein streams. Specific tasks include investigating catalytic upgrading of the lipid stream, biochemical conversion of the carbohydrates to biofuels, and supply of the protein to Sandia National Laboratories for upgrading to butanol. NREL is also testing the physical and chemical properties of the novel fuels and fuel intermediates with respect to compatibility with existing fuel infrastructure. In this way, we will develop a conversion process that optimizes yields and reduces production costs.

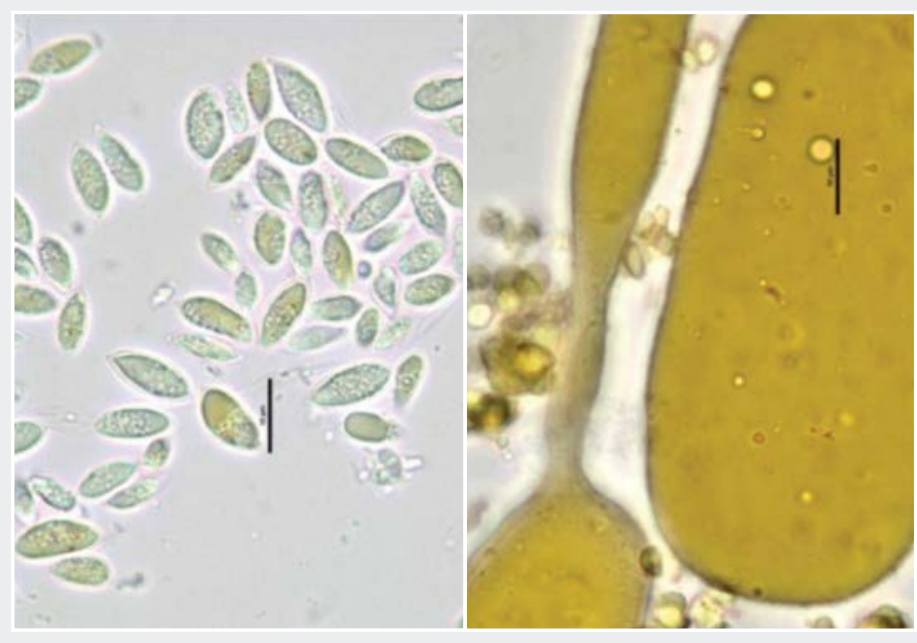

Physical appearance of algae before and after biochemical conversion, showing large oil droplets formed during the reaction. Photo by Nicholas Sweeney, NREL 


\section{Algae Testbed Public Private Partnership (ATP³)}

To accelerate the commercialization of technologies based on algal biofuels, NREL is part of a team led by Arizona State University to fill data gaps for algal cultivation and processing and to provide access for technology developers to its five testbed sites. NREL leads the Integration and Analysis directorate, which includes oversight of experimental design, data management, techno-economic analysis, and analytical method harmonization.

\section{Optimization of Biogas Production from Algal Residues via Anaerobic Digestion}

NREL, together with Washington State University, is investigating the optimization of biogas production from algal residues via anaerobic digestion. In this project, research is being carried out to maximize biogas yields and to understand effluent treatment requirements and the fate and bio-availability of nitrogen and phosphorus (for recycle to algae cultivation) following anaerobic digestion.

\section{Development of Robust and High-Throughput Characterization Technologies}

NREL researchers are developing technologies for the analysis of biofuel process-relevant components in algal biomass. These methods will allow researchers to assess the efficiency of the

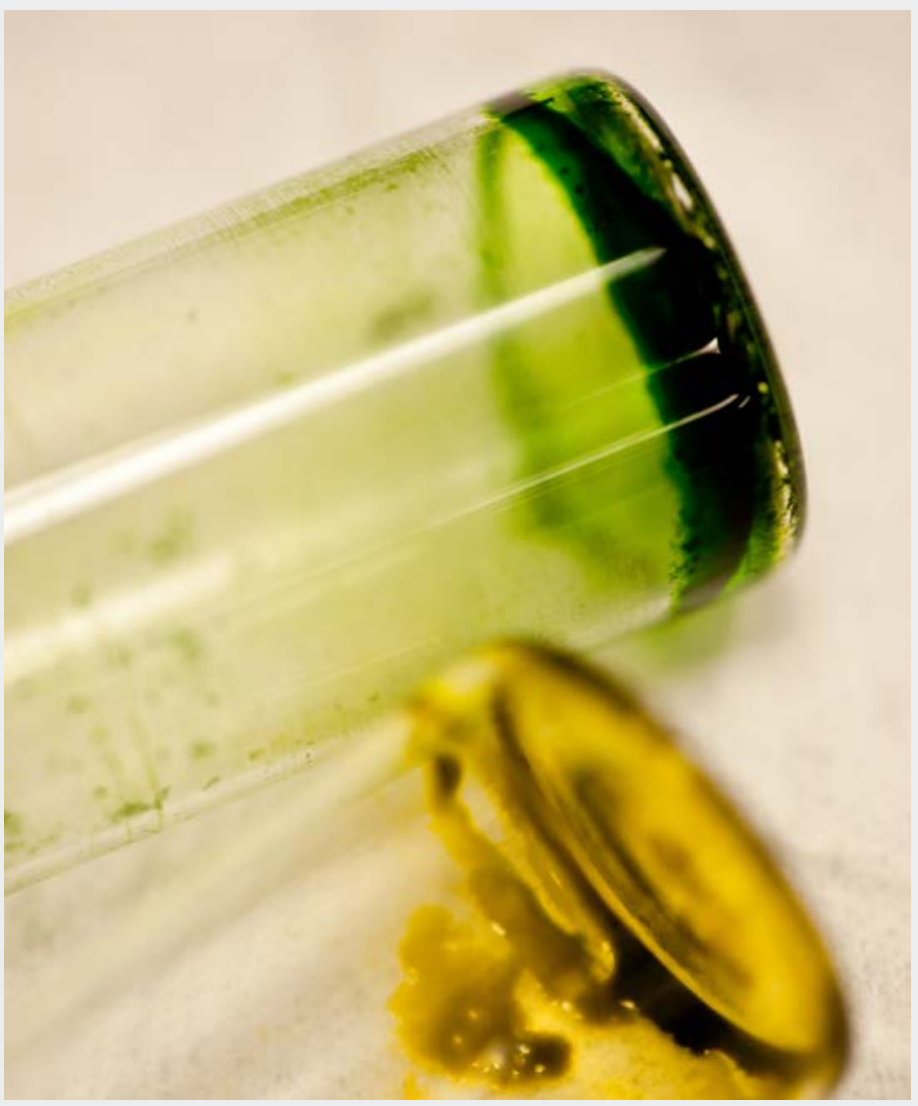

Extracted algae oil. Photo by Dennis Schroeder, NREL 18230 overall algal biofuels production process through the use of techno-economic analyses. This will keep NREL at the forefront of compositional analysis of novel feedstocks and allow for accurate quantification of important constituents in the process. In addition, scientists are also developing high-throughput spectroscopic-prediction methods to rapidly measure the composition of algal biomass from model organisms and culture collection strains in minutes rather than days.

\section{Techno-Economic Assessment of Algal Biofuels Processes}

NREL supports DOE's efforts to establish cost targets and track progress toward meeting these targets through techno-economic modeling and analysis of biofuel production pathways. These models are leveraged to quantify R\&D efforts from an economic standpoint and to provide peer-reviewed documentation of projected algal biofuel production costs. Additionally, several DOE-funded projects, including consortia like the National Alliance for Advanced Biofuels and Bioproducts (NAABB), Sustainable Algal Biofuels Consortium (SABC), and $\mathrm{ATP}^{3}$, are studying the viability of algae as a biofuels feedstock in the context of resource availability and techno-economic barriers and opportunities. The NREL team provides data for the modeling of the process and final fuel costs.

\section{Establishment of a 400+ Bioenergy-Focused Microalgae Strain Collection Using Rapid, High-Throughput Methodologies}

NREL, the Colorado School of Mines, and the National Research Council of Canada have established a bioenergy focused strain collection. High-throughput technologies were used to discover new novel microalgae strains that can be used for biofuel and bioproduct applications. More than 400 microalgal strains have been generated using novel strain isolation methods and a culture collection that was screened and catalogued, and isolates with high-growth and lipid-accumulation properties were cultivated at larger scales. The culture collection spans strains from the southwestern United States to isolates from northern latitudes,

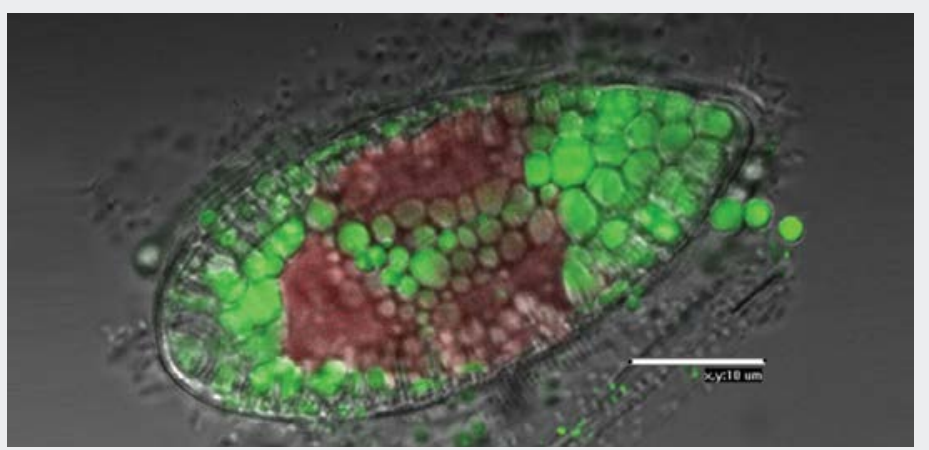

High lipid strain from NREL culture collection. Photo by Lee Elliott, Colorado School of Mines 
including marine, fresh-water, and hyper-saline environments. These projects were funded by the Colorado Center for Biorefining and Biofuels and DOE.

\section{Molecular Foundations of Algal Biofuel Production: Proteomics and Transcriptomics of Algal Oil Production}
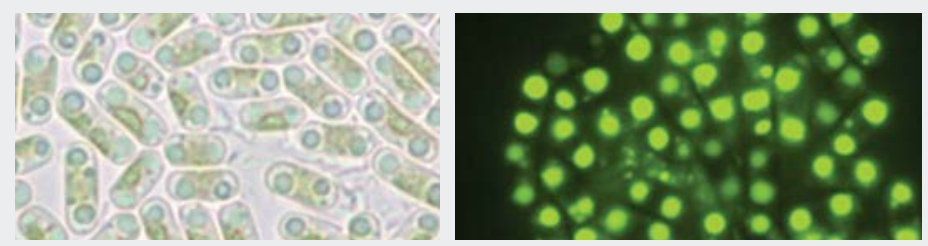

Examples of high lipid isolates from NREL culture collection. Photo by Lee Elliott, Colorado School of Mines

\section{Nitrogen Replete}

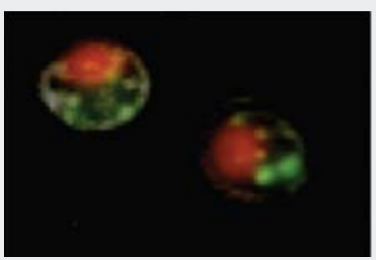

\section{Nitrogen Deplete}

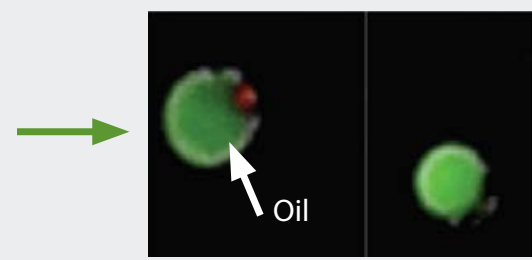

Increase of lipid content in Chlorella vulgaris with nitrogen starvation. Photo by Michael Guarnieri, NREL

NREL is combining a proteomics and transcriptomics approach to fully characterize the molecular foundation of algal biofuels production with external environmental conditions in oil accumulating microalgae. The combined '-omics' approach will lead to a better understanding of the changes that occur during transition to high-lipid growth conditions. These technologies provide NREL with information that can be used to identify genes and pathways involved in biofuel production and to guide strain improvement strategies.

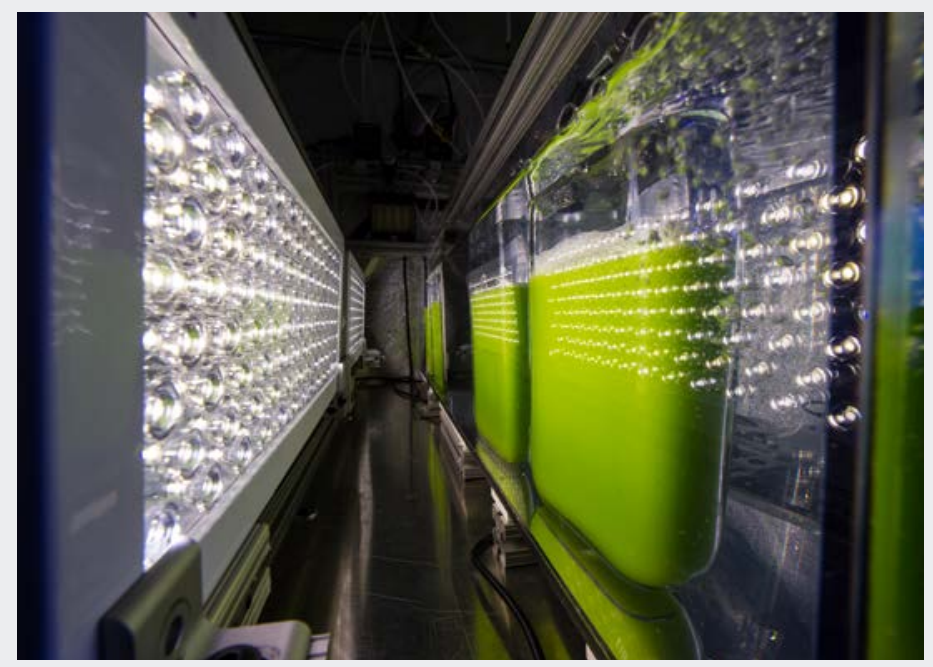

Photobioreactor used for carbon uptake and composition modeling. Photo by Dennis Schroeder, NREL 25505

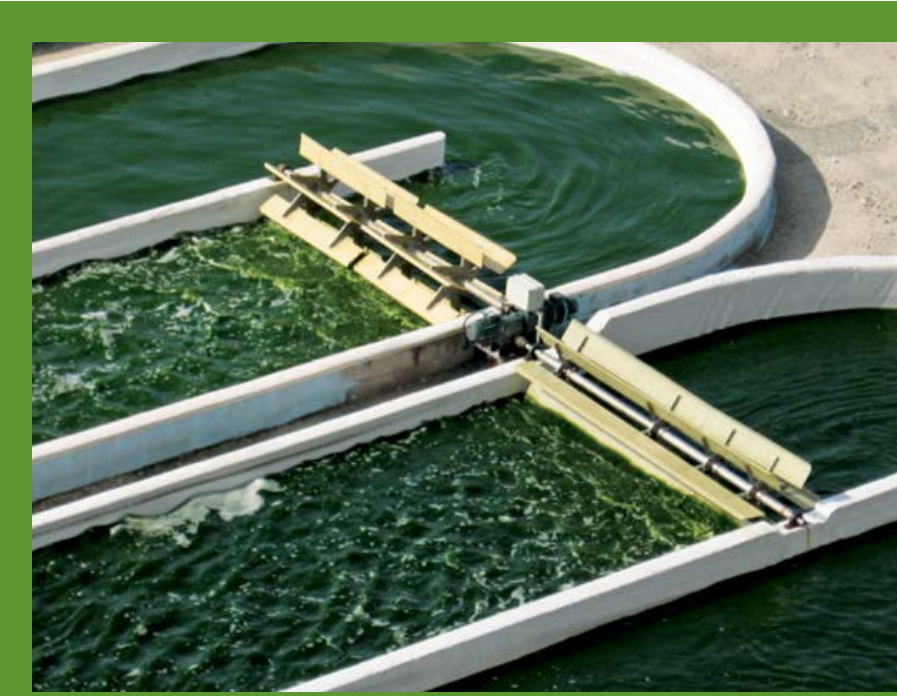

Photo by Nature Beta Technologies Ltd, Ei, NREL 22155

Sheehan et al. (1998) presaged the current revival in the field of algal biomass, concluding the DOE Algal Species Program close-out report with the following:

"When the time is right, we fully expect to see renewed interest in algae as a source of fuels and other chemicals."

\section{Investigation of Cell Signaling Mechanisms Governing Lipid Accumulation}

Scientists in NREL's Applied Biology section are investigating cell cycle and cell signaling mechanisms governing nutrient deprivation-induced lipid accumulation. This work is funded through NREL's LDRD program, with the goal of identifying and engineering gene targets for rapid induction of microalgal lipid accumulation in the absence of exogenous triggers. This approach has the potential to dramatically decrease costs associated with current algal cultivation strategies.

\section{Controlled Cultivation for Carbon Uptake and Composition Modeling}

NREL has a state-of-the-art photobioreactor system that allows for simulated cultivation of algae under mimicked outdoor conditions while monitoring $\mathrm{CO}_{2}$ assimilation, nutrient uptake rates, and full biochemical composition of the resulting biomass. 


\section{Publications}

Gerken, H. G., et al. (2013). "Enzymatic Cell Wall Degradation of Chlorella vulgaris and Other Microalgae for Biofuels Production." Planta (237:1) pp. 239-253.

Guarnieri, M.T., et al. (2013). "Proteomic analysis of Chlorella vulgaris: Potential targets for enhanced lipid accumulation." J Proteomics. DOI: 10.1016/j.jprot.2013.05.025 (Epub ahead of print).

Pienkos, P.T., et al. (2013). "The Energy-Water-Food Nexus Through the Lens of Algal Systems." Industrial Biotechnology (9:4); pp. 158-162. DOI:10.1089/ind.2013.1579.

Davis, R., et al. (2012). "Renewable Diesel from Algal Lipids: An Integrated Baseline for Cost, Emissions, and Resource Potential from a Harmonized Model.” ANL/ESD/12-4, NREL/TP-510055431, PNNL-21437. www.nrel.gov/docs/fy12osti/55431.pdf

Elliott, L.G., et al. (2012). "Establishment of a Bioenergy-Focused Microalgal Culture Collection." Algal Research. DOI: 10.1016/j. algal.2012.05.002.
Laurens, L. M.L., et al. (2012). “Algal Biomass Constituent Analysis: Method Uncertainties and Investigation of the Underlying Measuring Chemistries." Analytical Chemistry (84:4); p. 1879.

Guarnieri, M., et al. (2012). "Complex System Engineering: A Case Study for an Unsequenced Microalga." Engineering Complex Phenotypes in Industrial Strains. Patnaik, R., ed. John Wiley \& Sons, Inc.

Pienkos, P.T., et al. (2011). "Making Biofuel from Microalgae." American Scientist (99:6); p. 474. DOI: 10.1511/2011.93.474.

Guarnieri, M.T., et al. (2011). "Examination of Triacylglycerol Biosynthetic Pathways via De Novo Transcriptomic and Proteomic Analyses in an Unsequenced Microalga." PLOS ONE, (6:10); e25851.

Davis, R., et al. (2011). "Techno-Economic Analysis of Autotrophic Microalgae for Fuel Production." Applied Energy. (88:10). pp. 3524-3531.

\section{Contacts}

Philip Pienkos, Principal Group Manager

Analytical Chemistry and Life Sciences

National Bioenergy Center

Philip.Pienkos@nrel.gov

www.nrel.gov/biomass/microalgal_biofuels.html

NREL is a national laboratory of the U.S. Department of Energy Office of Energy Efficiency and Renewable Energy Operated by the Alliance for Sustainable Energy, LLC

NREL/FS-5100-60483 • September 2013 